\title{
How to fight with heart diseases
}

\begin{abstract}
The 2010 dietary cholesterol recommendations were derived from guidelines established in the 1960s, when little scientific evidence was present, other than the possible association between saturated fat and dietary cholesterol, as well as animal studies, in which cholesterol was fed in amounts that exceeded typical or normal intakes. Aim of this research was to compare individual and combined effects of Ispaghola and Anjeer in primary as well as secondary hyperlipidemia. It was single blind placebo-controlled research work, conducted from January 2017 to April 2017 at General Hospital, Lahore, Pakistan. One hundred male and female hyperlipidemic patients were selected for research, age range from 20 to 65 years. Inclusion criteria were primary, secondary hyperlipidemic patients. Written and explained consent was taken from all participants and approved by research ethics committee of the hospital. Patients were divided in four equal groups, comprising 25 patients in each group. Their baseline systolic/diastolic blood pressure and lipid profile was estimated and kept in individual's folder. Group-I was on Ispaghula 10 grams, group-II was on 10 grams Anjeer, and group-III was advised to take 10 grams Ispaghula and 10 grams Anjeer in combination for 3 months. Group-IV was on placebo therapy. After 3 months when results were compiled and analyzed statistically, it was observed that Ispaghula decreased LDL-c significantly with p-value $<0.05$, Anjeer decreased LDL-c significantly with $\mathrm{p}$-value $<0.01$. When both herbs were combinely given, they reduced systolic BP with p-value $<0.01$, LDL-c reduced highly significantly with p-value $<0.001$, and HDL-c increased significantly with $\mathrm{p}$-value $<0.05$. It was concluded from the study that Ispaghula and Anjeer have LDL-c lowering potential if given separately. When used in combination they reduce systolic BP, LDL-c, and increase HDL-c.
\end{abstract}

Volume 12 Issue I - 2019

\author{
Azmat Ali AP,' Shah Murad, ${ }^{2}$ Adnan Shafique, ${ }^{3}$ \\ Ejaz Fatima ${ }^{4}$ \\ 'Pharmacology at Alnafees Medical College, Islamabad Pakistan \\ ${ }^{2}$ Professor and HOD of Pharmacology, IMDC, Pakistan \\ ${ }^{3}$ Consultant Pharmacist at MCC Pharmacy, Islamabad, Pakistan \\ ${ }^{4}$ Associate Professor of Pharmacology, LMDC, Pakistan
}

Correspondence: Shah Murad, Department of Pharmacology,

Akbar Niazi Teaching Hospital, Islamabad Medical and Dental

College, Pakistan, Tel +923।422434I5,

Emailshahhmurad65@gmail.com

Received: November 23, 2018 | Published: February 21, 2019

Keywords: High serum fats, atherosclerosis, dietary fibers, pakistani fruits

\section{Introduction}

Heart disease is the result of the narrowing of the arteries that supply the heart with blood, oxygen and nutrients. This process, called coronary artery disease, can generally be traced to a condition called atherosclerosis, the build-up of cholesterol-rich fatty deposits, or plaques, on the inside of arterial walls. ${ }^{1}$ As these deposits accumulate over time, the coronary arteries narrow to the point that the flow of oxygenated blood to the heart is impeded. (Arterial spasms - triggered by smoking, extreme emotional stress, or exposure to very cold temperatures - may also cause coronary arteries to narrow suddenly and dangerously.) $)^{2}$ Since the US dietary cholesterol recommendations were first promulgated, a large number of longitudinal observational studies and intervention trials have been published on the relation between cholesterol intake and cardiovascular outcomes. Some studies have reported dietary cholesterol to increase CVD risk, whereas others reported a decreased risk or no change with higher cholesterol intake. ${ }^{3,4}$ Given these inconsistencies among studies, the purpose of this systematic review is to determine the potential effect of dietary cholesterol on incident CVD and on serum concentrations of total cholesterol, LDL cholesterol, HDL cholesterol, LDL to HDL ratio, and triglycerides in adults. ${ }^{5}$ Secondary and primary hyperlipidemia cause LDL-oxidation, leading to formation of atherosclerotic plaques which get deposited at interior wall of coronary vessels, making it narrow and causing, hypertension, CCF, cardiac arrhythmias, and cardiac arrest. ${ }^{4-6}$ To decrease incidences of advanced heart diseases, it is necessary to leave sedentary life, over consumption of alcohol, cigarette smoking, to reduce body weight. ${ }^{7}$ Allopathic drugs used in hyperlipidemia have unwanted effects on human body, so herbs can be safe to use in prevention and treatment of primary as well as secondary hyperlipidemia. ${ }^{8}$ Psyllium hydrophilic muciloid or Ispaghola inhibit enterohepatic circulation of bile and excreted it out through feces, thus causing hepatocytes to form bile instead of cholesterol. ${ }^{9-13}$ Anjeer of figs contain various active ingredients which are antioxidant in nature. Phenolic compounds of Anjeer have also potential to scavenge free radicals formed in metabolic processes in human body. Various minerals and vitamins present in Anjeer cause transcription of amino acids which lead to hepatocytes to synthesize apoproteins, essential for structural and functional integrity of HDL in human body. Figs reduce the amount of triglycerides in the blood. Triglycerides are the culprit for inducing heart disease as they are fat particles which accumulate along the blood vessel and cause heart attacks..$^{14}$

\section{Patients and methodology}

It was single blind placebo-controlled research work, conducted from January 2017 to April 2017 at General Hospital, Lahore, Pakistan. One hundred male and female hyperlipidemic patients were selected for research, age range from 20 to 65 years. Inclusion criteria were primary, secondary hyperlipidemic patients. Exclusion criteria was patients suffering from any chronic illness like CCF, cardiac arrhythmias, thyroid disease, peptic ulcer, Alzheimer's disease, major depression, and renal or hepatic impairment. Written and explained consent was taken from all participants and approved by research ethics committee of the hospital. Patients were divided in four equal groups, comprising 25 patients in each group. Their baseline determination of LDL-cholesterol and HDL-cholesterol was made at Biochemistry laboratory and was kept in specially formatted Performa. Group-I was advised to take dried Figs 10 grams daily in three equally divided doses. Group-II was advised to take 10 grams of Psyllium husk daily in three divided doses before each meal for three months. Group-III was advised to take 10 grams Figs and 10 grams Psyllium husk in three divided doses daily for three months. GroupIV was advised to take one capsule (containing grinded wheat) with each meal time, thrice daily for the period of three months. They were 
advised to come for follow up monthly. Their LDL-cholesterol and HDL-cholesterol, blood pressure was measured/determined monthly. After three months all parameters were evaluated like their blood pressure and lipid profile. Results were compiled and statistically analyzed by using SPSS version 5.02014 . Mean values of the tested parameters were expressed with \pm SEM and paired ' $t$ ' test was applied to determine significant changes in mean values before and after treatment. P-value $>0.05$ was considered as non-significant change, p-value $<0.01$ was considered as significant and p-value $<0.001$ was considered as highly significant change in tested parameters.

\section{Results}

When results were compiled and statistically analyzed, it was observed that Ispaghula reduced SBP of 23 patients from $130.11 \mathrm{~mm}$ of hg to $125.89 \mathrm{~mm}$ of hg, and 89.45 to $87.91 \mathrm{~mm}$ of hg in systolic and diastolic BP respectively. Both are non significant results. 9.06 $\mathrm{mg} / \mathrm{dl}$ decrease in LDL-c was observed which significant change is. HDL-c was increased from $38.43 \pm 1.65$ to $41.87 \pm 1.95 \mathrm{mg} / \mathrm{dl}$ which is non-significant change in the parameter. Anjeer decreased 133.55 to $130.06 \mathrm{~mm}$ of hg and 91.65 to $85.96 \mathrm{~mm}$ of $\mathrm{hg}$ in systolic and diastolic BP respectively and change in both parameters are nonsignificant. This fruit reduced LDL-c from 175.88 to $159.20 \mathrm{mg} /$ $\mathrm{dl}$ in 25 hyperlipidemic patients which is significant change in the parameter. HDL-c increased from 33.86 to $39.01 \mathrm{mg} / \mathrm{dl}$ which is nonsignificant change. When both agents were used combine SBP, and DBP were decreased from 134.76 to 121.88 and 90.49 to $85.02 \mathrm{~mm}$ of hg in 25 patients. Change in systolic BP is significant but in diastolic $\mathrm{BP}$ it is non-significant change. LDL-c in this group was reduced from 178.76 to $160.99 \mathrm{mg} / \mathrm{dl}$ which is highly significant change in the parameter. HDL-c was increased from 39.61 to $48.69 \mathrm{mg} / \mathrm{dl}$ which is significant change. In placebo group changes in all parameters are non-significant (Table 1).

Table I Showing pre and post-treatment mean values, \pm sem, changes in results and biostatistical significance

\begin{tabular}{|c|c|c|c|c|c|c|}
\hline Group of patients & $\begin{array}{l}\text { Evaluated } \\
\text { parameters }\end{array}$ & $\begin{array}{l}\text { Pre-treatment } \\
\text { values }\end{array}$ & $\begin{array}{l}\text { Post-treatment } \\
\text { values }\end{array}$ & $\begin{array}{l}\text { Change in pre and } \\
\text { post-treatment } \\
\text { values }\end{array}$ & $\begin{array}{l}\text { Change in } \\
\text { percentage }\end{array}$ & p-value \\
\hline \multirow{4}{*}{ Ispaghula $n=23$} & SBP & $130.11 \pm 2.01$ & $125.89 \pm 2.00$ & 4.22 & 3.24 & $>0.05$ \\
\hline & DBP & $89.45 \pm 1.98$ & $87.91 \pm 2.90$ & 1.54 & 1.72 & $>0.05$ \\
\hline & LDL-c & $179.04 \pm 2.18$ & $169.98 \pm 2.76$ & 9.06 & 5.06 & $<0.05$ \\
\hline & HDL-c & $38.43 \pm 1.65$ & $41.87 \pm 1.95$ & 3.44 & 8.95 & $>0.05$ \\
\hline \multirow{4}{*}{ Anjeer $n=25$} & SBP & $133.55 \pm 1.75$ & $130.06 \pm 1.09$ & 3.49 & 2.61 & $>0.05$ \\
\hline & DBP & $91.65 \pm 1.54$ & $85.96 \pm 2.02$ & 5.69 & 6.2 & $>0.05$ \\
\hline & LDL-c & $175.88 \pm 2.67$ & $159.20 \pm 2.66$ & 16.68 & 9.48 & $<0.01$ \\
\hline & HDL-c & $33.86 \pm 1.74$ & $39.01 \pm 2.64$ & 5.15 & 15.2 & $>0.05$ \\
\hline \multirow{4}{*}{$\begin{array}{l}\text { Ispaghula }+ \text { Anjeer } \\
\mathrm{n}=25\end{array}$} & SBP & $134.76 \pm 1.88$ & $121.88 \pm 2.76$ & 12.88 & 9.55 & $<0.01$ \\
\hline & DBP & $90.49 \pm 2.63$ & $85.02 \pm 3.96$ & 5.47 & 6.04 & $>0.05$ \\
\hline & LDL-c & $178.76 \pm 3.12$ & $160.99 \pm 3.86$ & 17.77 & 9.94 & $<0.001$ \\
\hline & HDL-c & $39.61 \pm 2.61$ & $48.69 \pm 3.63$ & 9.08 & 22.92 & $<0.05$ \\
\hline \multirow{4}{*}{ Placebo $n=24$} & SBP & $120.05 \pm 1.87$ & $119.77 \pm 2.91$ & 0.28 & 0.23 & $>0.05$ \\
\hline & DBP & $75.85 \pm 2.91$ & $75.01 \pm 2.77$ & 0.84 & 1.1 & $>0.05$ \\
\hline & LDL-c & $141.05 \pm 1.97$ & $140.25 \pm 2.22$ & 0.8 & 0.56 & $>0.05$ \\
\hline & HDL-c & $42.56 \pm 2.06$ & $42.59 \pm 1.64$ & 0.03 & 0.07 & $>0.05$ \\
\hline
\end{tabular}

Key: All parameters and change before and after treatment in parameters are measured in mg/dl and their mean values are written with $\pm \mathrm{SEM}$. P-value $<0.0 \mathrm{I}=$ significant, $\mathrm{P}$-value $>0.05=$ non-significant changes in lipid profile. HDL-c and LDL-c values are measured in milligrams per milliliter. SBP stands for systolic blood pressure, DBP stands for diastolic blood pressure and were measured in millimeter of mercury (HG). n, sample size.

\section{Discussion}

Recent evidence suggests that even moderate-intensity activity, including brisk walking, is associated with substantial reduction of cardiovascular disease risk. These findings support the 1995 federal exercise guidelines endorsing 30 minutes of moderately intense physical activity most days of the week, a program that should be feasible and safe for most of the population. Regular exercise and maintenance of healthy weight should also help reduce insulin resistance and the risk of non-insulin-dependent diabetes mellitus, which appears to be an even stronger risk factor for cardiovascular disease in women than in men. Diabetes is associated with a threefold to sevenfold elevation in cardiovascular disease risk among women, compared with a twofold to threefold elevation among men. Approximately half of all deaths in patients with non-insulin dependent diabetes mellitus are due to heart disease. Ispaghula (psyllium husk) has hypolipidemic effects due to its inhibition of enterohepatic circulation of bile acids in GIT. Decreased pool of bile in gall bladder causes hepatocytes to synthesize bile instead of cholesterol in liver. Anjeer or Figs are well known for their antioxident properties and they contain phytochemicals which cause transcriptory synthesis of apoproteins which are structural and physiological parts of HDL. Both of these characteristics have proved Anjeer as hypolipidemic herbs/fruits. Flavonoids, fluranocoumarins, organic acids found in 
these fruits have hypolipidemic features as mentioned by Guruj Y et al. ${ }^{15}$ In our observation Ispaghula reduced LDL-c in 23 patients which is significant when analyzed statistically. Ispaghula's effects in other parameters are very mild and non-significant. These results match with results of study conducted by Marstein $\mathrm{M}$ et al., ${ }^{16}$ who did observe almost same changes in LDL-c when 30 grams of ispaghula was used for one month in 30 hyperlipidemic patients. Weildshr G et al. ${ }^{17}$ explained MOA of Ispaghula as hypolipidemic agent that husk of these seeds inhibit enterohepatic circulation of bile causing synthesis of bile instead of cholesterol by hepatocytes. Schooler $\mathrm{R}$ et al. ${ }^{18}$ explained that HDL-c must be increased when too much psyllium husk may be used in remarkable time period, but sample size also counts, they further explained. Jeajath $\mathrm{C}$ et al. ${ }^{19}$ stated that apoproteins formation in liver cells is most important step induced by psyllium husk as hypolipidemic agent. Anjeer when used alone in 25 hyperlipidemic patients in our observation are effective to reduce LDL-c only, as other parameters proved no significant changes. It was also proved by study conducted by Restham T et al. ${ }^{20}$ They proved 13 $\%$ decrease in LDL-c in 53 hyperlipidemic and hypertensive patients when they used 10 grams anjeer for four months. Jufthas $\mathrm{Y}$ et al. ${ }^{21}$ wrote that environmental factors affects study results when sample is only taken from human population. Close observation and frequent follow up visits are needed and necessary steps in human involved research work. Astolth ER et al. ${ }^{22}$ advised their patients for strict half an hour physical exercise and banned them to take any processed and dairy foods during their drug trial. They also got same results of anjeer fruit in hyperlipidemic and hypertensive patients. Ethere $\mathrm{C}$ et al. ${ }^{23}$ used fruit extract of anjeer in albino rats and proved that HDL-c may be affected significantly. These results explain close observatory environment for avoiding any false data/results during any sensitive research work. In our research it was proved that combination of anjeer and psyllium husk affect on systolic BP, LDL-c and HDL-c, but diastolic BP is not affected significantly. Thyswe JE et al. ${ }^{24}$ proved biostatistically significant effects of psyllium seeds and anjeer on LDL-c, HDL-c, TG, TC, VLDL in diet-induced hyperlipidemic mice. Again it is proved and strongly recommended that controlled environment is first necessary step to get true results from research work. Jouhall C et al., ${ }^{25}$ Ersuwe T et al., ${ }^{26}$ Thooharr F et al., ${ }^{27}$ Rothr E et al. ${ }^{28}$ Usde $\mathrm{E}$ et al. ${ }^{29}$ mentioned that in human body just to reduce lipid levels can prevent development of CAD, heart arrhythmias, $\mathrm{CCF}$, and cardiac arrest.

\section{Conclusion}

It was concluded from this research work that fruits like anjeer and fibers like psyllium can work to reduce serum lipids in human body and because of prevention from complications of hyperlipidemia.

\section{Acknowledgments}

We acknowledge all types of help and cooperation by management of General Hospital Lahore to conduct this research work.

\section{Conflicts of interest}

There is no conflict of interest regarding this research work with any institution or organization or individual.

\section{Funding}

There is no conflict of interest regarding this work with any party/ ORG or institution.

\section{References}

1. Gunhgarr T, Fudaat T. Complications of Hyperlipidemia. Jou Ir Med. 2013;4(1):111-118.

2. Welkr R, Swath RT, Mekran FR. Beyond the history: Hyperlipidemia and Antioxidants. Cl Jou Nutr Med. 2014;6(7):45-47.

3. Therome E, Jethusa MM, Mattak GG, Fuller CV. Preventable factors for CAD. Jou Cl Med. 2014;8(3):122-126.

4. RE putrkr, SC Johnson, TY Mokrann. Prevention is better than tretment in heart diseases. JCHD. 2013;4(4):22-29.

5. Ethraq E, Javlase Y, Laguerre M, et al. Medications used in oxidative stress. Ir J Med Sc. 2012;6(2):61-67.

6. Forsat RR, Miller NJ, Paganga TG. Herbs in general considerations. HHJ. 2012;6(3):44-49.

7. Kostapanos MS, Liamis GL, Milionis HJ, et al. Do statins beneficially or adversely affect glucose homeostasis? Curr Vasc Pharmacol. 2010;8:612-631.

8. Mukkarj RT, Rusat VB. Use of Herbs in Variuous Ethnic Groups. Ethan Bot J. 2012;15(7):777-779.

9. Desortin J, Ulharv R. New approaches to foods and heart diseases. $J$ Nutr. 2013;501-504.

10. Malker T. Enterohepatic circulation and bile. GIT Jou. 2012;6(4):222-228.

11. Jahily C, Pitwat F. Bile acid binding resins and healthy heart. Vas Jou Is. 2014;4(2):200-204.

12. Lauja V, Herrth U, Yulr B, et al. Clean your gastrointestinal tract and remain healthy. Health \& Nutr. 2012;6(4):222-225.

13. C Peraze, T Mughwann. Hyperlipidemia, Psyllium husk and Heart. New Jou Vas Med. 2012;3(6):344-347.

14. Kunal S, Sidhart T, Parkash A. Ficus carica and heart diseases. Eth Bot. 2013;4(5):77-90.

15. Guruj Y, Sheloww E, Thermaw T, et al. Phytochemistry of ficus carica. Phyt J. 2014;12(5):555-557.

16. Marstein M, Ythfaw C, Hurrha J. 30 grams psyllium for one month for cure of atherogenesis. Vas J Med. 2015;4(7):333-337.

17. Weildshr G, Helmoy T, Welkr J, et al. Mode of action of Ispaghola. Eth Jou Bot. 2012;5(5):122-127.

18. Schooler R, Thupase T, Thymosrr F, et al. Effects of psyllium on lipid profile. Lipids J. 2015;6(7):222-226.

19. Jeajth C, Hermou V, Kulhar Y, et al. Apoproteins and blood lipids. Cardio J Ir. 2013;6(7):612-615.

20. Restham T, Buldow E, Molakt V. Interaction of bad cholesterol with ficus carica. Ann SMC. 2015;6(5):100-105.

21. Jufthas Y, Lamaj V, Helkaar C, et al. Research protocols and methodologies. NJDDT. 2016;2(6):12-17.

22. Astolth ER, Yuthmas DD, Fituhar VR. How to deal with lipids research? JCNM. 2012;6(1):19-23.

23. Ethere C, Yethma N, Melojaw V, et al. Use of Ficus carica in albino rats. Egy J Cl HSc. 2011;7(6):456-459.

24. Thyswe JE, thymolov YR, Teetehar RR. Use of herbs for normalizing blood lipids. Cl Res J. 2016;6(8):88-90.

25. Jouhall C, Welker T, Ethere C, et al. Treatment of diabetes, hypertension, and hyperlipidemia by herbs. Herb Med J. 2014;6(6):578-581. 
26. Ersuwe T, Therov J, Ursita Y, Nelaja R. Combination therapy by fruits and fibers. JMSR. 2014;23(8):89-92.

27. Thooharr F, Helhar T, Merdhat E, et al. How to cobate hyperlipidemia Jou Etho Med Colllg. 2012;13(8):456-459.
28. Rothr E, Wernav F, Juhfr D, et al. Ethanobotnical approaches in therapeutics. JEMC. 2014;17(8):122-127.

29. Usde E, Dekhta V, Hesko B, et al. Complications of alternative therapies. JAMU. 2016;7(8):99-106. 